\title{
An Attempt to Standardize Kurjak's Antenatal Neurodevelopmental Test: Osaka Consensus Statement
}

\author{
${ }^{1}$ Milan Stanojevic, ${ }^{2}$ Amira Talic, ${ }^{1}$ Berivoj Miskovic, ${ }^{1}$ Oliver Vasilj, ${ }^{3} \mathrm{Afaf}$ Naim Shaddad, ${ }^{3}$ Badreldeen Ahmed \\ ${ }^{4,5}$ Aida Salihagic Kadic, ${ }^{4}$ Maja Predojevic, ${ }^{6}$ Radu Vladareanu, ${ }^{6}$ Daniela Lebit, ${ }^{3}$ Salwa Abu-Yaqoub \\ ${ }^{7}$ Madeeha Al-Noobi \\ ${ }^{1}$ Department of O bstetrics and Gynecology, Medical School University of Zagreb, Sveti Duh Clinical Hospital, Zagreb, Croatia \\ ${ }^{2}$ Department of Obstetrics and Gynecology, University of Medical Sciences and Technology, Academy Charity Hospital, Khartoum, Sudan \\ ${ }^{3}$ Hamad Medical Corporation, Doha, Qatar \\ ${ }^{4}$ Department of Physiology, Medical School University of Zagreb, Zagreb, Croatia \\ ${ }^{5}$ Croatian Institute for Brain Research, Medical School University of Zagreb, Zagreb, Croatia \\ ${ }^{6} \mathrm{C}$ arol Davila University of Medicine, Bucharest, Romania \\ ${ }^{7}$ Head, Department of Radiology, Armed Forces Medical Services, Doha, Qatar
}

Correspondence: Milan Stanojevic, Department of Obstetrics and Gynecology, Medical School University of Zagreb, Sveti Duh Clinical Hospital, S veti Dub-64, 10000 Zagreb, Croatia, Phone: ++38513712316, ++38513745534, e-mail: milan.stanojevic@ optinet.hr

\section{ABSTRACT}

Analysis of the dynamics of fetal behavior in comparison with morphological studies has led to the conclusion that fetal behavioral patterns are directly reflecting developmental and maturational processes of fetal central nervous system (CNS). Four-dimensional ultrasound (4D US) offers a practical means for assessment of both the brain function and structure. The visualization of fetal activity in utero by 4D US could allow distinction between normal and abnormal behavioral patterns which might make possible the early recognition of fetal brain impairment. That new technology enabled introduction of Kurjak's antenatal neurodevelopmental test (KANET) in low- and high-risk pregnancies. In order to make the test reproducible, the standardization of the test was proposed in Osaka, J apan, during the International Symposium on Fetal Neurology of International Academy of Perinatal Medicine.

The KANET should be performed in the 3rd trimester from 28th to 38th week of gestation. The assessment should last from 15 to 20 minutes, and the fetuses should be examined when awake. If the fetus is sleeping, the assessment should be postponed for 30 minutes or for the next day between 14 and 16 hours. In cases of definitely abnormal or borderline score, the test should be repeated every two weeks till delivery. New modified KANET test should be used with eight instead of 10 parameters: Facial and mouth movements are combined in one category, isolated hand movements and hand to face movements are combined in one category. The score should be the same for abnormal fetuses 0 to 5 , borderline score is from 6 to 13 and normal score is 14 or above.

After 4D US assessment of behavioral patterns in the fetuses from high-risk pregnancies, it is very important to continue with follow-up after delivery in infants who were borderline or abnormal as fetuses. Postnatal assessment of neonates includes initial neurological assessment according to Amiel-Tison's methodology (Amiel-Tison Neurological Assessment at Term, ATNAT) in the early neonatal period and every two weeks in preterm infants till discharge and at the postmenstrual age (PMA) between 37 and 40 weeks. If ATNAT is borderline or abnormal, initial assessment of general movements at the age of 36 to 38 weeks of PMA should be performed, than at writhing age (between 46 and 52 weeks), and at the fidgety age after 54 weeks of PMA. If the finding of fidgety movements is mildly abnormal or definitely abnormal, then one more assessment should be done in 2 to 4 weeks till PMA of 58 weeks. Brain ultrasonography should be performed in the first week of life and every 2 weeks afterward till discharge. In severely affected infants with grade 3 and above intraventricular hemorrhage, and those highly suspicious of hypoxic ischemic brain damage, magnetic resonance (MR) should be done if available. Infants should be followed until the age of at least 24 months when diagnosis of disabling or nondisabling cerebral palsy can be ultimately made. Infants with $C P$ should be reassessed at the age of 6 years.

Keywords: Fetal neurobehavior, 4D ultrasound, Neurobehavioral screening, Postnatal neurological screening, Cerebral palsy.

\section{INTRODUCTION}

A nalysis of the dynamics of fetal behavior in comparison with morphological studieshas led to theconclusion thatfetal behavioral patterns are directly reflecting developmental and maturational processes of fetal central nervous system (CNS). ${ }^{1-3}$ It has been suggested that theassessment of fetal behavior and developmental processes in different periods of gestation may make possible, the distinction between normal and abnormal brain development, as well as early diagnosis of various structural or functional abnormalities. ${ }^{4}$ Four-dimensional ultrasound (4D US) or realtime three-dimensional ultrasound (3D US) offers a practical means for assessment of both the brain function and structure. ${ }^{5}$ A dditionally, 4D US enables spatial observation of fetal face, which two-dimensional ultrasound (2D US) does not. In a relatively shortperiod of time, $4 \mathrm{D}$ US has stimulated multicentric studies on fetal and even embryonic behavior with more convincing imaging than conventional 2D US and nonultrasonic methods. The visualization of fetal activity in utero by $4 D$ US 
could allow distinction between normal and abnormal behavioral patterns, which mightmakepossibletheearly recognition of fetal brain impairment. ${ }^{6}$ That new technology enabled introduction of K urjak' santenatal neurodevel opmental test(K ANET) in lowand high-risk pregnancies in many centers. ${ }^{7}$ I norder to make the test reproducible, the standardization of the test was proposed in Osaka, J apan during the International Symposium on Fetal N eurology of I nternational A cademy of Perinatal M edicineheld from the 22nd to 24th 0 ctober, 2010. Session on Standardization in Fetal Neurology was chaired by A sim K urjak and M alcolm Levene, with the participation of many prominent scientists involved in prenatal and postnatal assessment of fetuses and infants with neurological risk ( $M$ anuel Carapato, M ilan Stanojevic, M alcolm Levene, A sim K urjak, Claudine A mielTison, J oseph Schenker, Hidenobu O hta, A ris A ntask lis, Zehra Nese Kavak, A ida Salihagic K adic, Birgit A rabin, Ritsuko K Pooh). A fter fruitful discussions, the standardization of the KANET was proposed and accepted.

The aim of this paper is to shortly present knowledge on 4D US imaging of fetal motoric activity throughout the pregnancy and to present the data obtained by application of KANET in low- and high-risk pregnancies. The Osaka consensus on standardization of KANET test will be presented in order to introduce K A NET into everyday clinical practice as reproducible and sensitive prenatal screening neurological test.

\section{FETAL BEHAVIOR}

Fetal behavior could be defined as any fetal action or reaction that could be observed by the mother or other more objective method, as ultrasonography or actocardiotocography. ${ }^{1,8,9}$ Although more than 100 years of curiosity in fetal behavior and al most three decades of sustained awareness and research, the study of fetal behavior has achieved widespread acceptance in perinatal medicine. ${ }^{10}$ For centuries, maternal registration of fetal movements and obstetrician auscul tation of fetal heartbeats were the only methods of the follow-up of fetal well-being in utero. It has been shown that fetal activity occurs as early as the late embryonic period, which is far earlier than the mother can sense it. ${ }^{8}$ Introduction of real-time ultrasound has been a turning point in the assessment of fetal behavior because it changed the definition of fetal behavior as any fetal activity observed or recorded with ultrasound equipment. ${ }^{1} \mathrm{~A} s$ it is not yet possible to assess functional development of the CNS directly, investigators have started to analyze fetal behavior in order to assess neurological maturation, including properties of fetal hemodynamics and the muscular system as well. ${ }^{11} \mathrm{~T}$ his technique al lowed the investigation of spontaneous fetal motor activity in utero. For the first time, studies of spontaneous prenatal movements and behavior in uterowere performed and published. Since fetal body movements give important information about the condition of the fetus, their quantitative as well as qualitative aspects were analyzed. ${ }^{12,13}$
There is a growing pool of evidence that many neurological disorders originate from prenatal rather than from postnatal period. ${ }^{3}$ A large spectrum of neurological problems, such as attention deficit hyperactivity disorder, schizophrenia, epilepsy or autism could result at least in part from prenatal neurodevelopmental problems. ${ }^{14}$ Furthermore, clinical and epidemiological studies have shown that even cerebral palsy (CP) most frequently results from prenatal rather than postnatal causes. ${ }^{15,16}$ A nalysis of the dynamics of fetal behavior has led to the conclusion that fetal behavioral patterns directly reflect developmental and maturational processes of the fetal CNS. These findings implicated that understanding the relation between fetal behavior and developmental processes in different periods of gestation would make possible the distinction between normal and abnormal development of the brain as well as the early diagnosis of various structural or functional abnormalities. ${ }^{3}$

\section{Fetal Behavior as an Indicator of Fetal Brain Impairment}

The integrity of fetal CNS could be assessed at least by two available methods. The first is assessment of previously described behavioral states, and the second is the qualitative assessment of general movements (GMs). ${ }^{17} \mathrm{M}$ aturation of behavioral states was show $n$ to be disturbed in risk pregnancies, like pregnancies with IUGR, diabetes mellitus or alcohol consumption. ${ }^{18}$ Even maternal emotions, like anxiety, influence the quantitative distribution of fetal behavioral states. ${ }^{19}$ The drawback is the unspecific nature of these changes in development limiting the clinical significance for predicting later impairment, which is in agreement with findings on abnormal states in the neonate. Qualitative and quantitative changes of the state variable are nonspecific sign of neural dysfunction and poor basis for prediction of later neurological outcome. ${ }^{19}$ State assessment can be used as an indicator of unspecific developmental delay. Q ualitative assessment of GM s was underestimated for a long time due to the 'subjectivity' of method which includes the observation of fetal movements. ${ }^{19}$ For many years, the interest of obstetricians was focused on the quantity of fetal movements which was considered as an indicator of fetal well-being. ${ }^{19} \mathrm{~L}$ ater studies have shown that count of movements is poor indicator of brain damage, mainly due to the great intra- and interindividual differences and the large overlap between normal and abnormal, which makes this method clinically useless. ${ }^{19}$ On the contrary, changes of the elegance and fluency, as well as the variability and fluctuation of intensity and speed of GM s, were shown to be prominent in the sick preterm infants. ${ }^{19}$ It became evident that the qual itative changes in GMs of both the fetus and the neonate precede quantitative changes when the integrity of the nervous system is impai red. ${ }^{19}$ Obviously, it is more difficult to objectify complex qualitative changes of GMs than simply to count them when they occur. The Gestalt-perception, which is term, was 
introduced by Prechtl as the overall impression on the quality of GMs, is excellent tool for dealing with such phenomenon. ${ }^{19}$ A $n$ important step is video recording of sufficient length of GMs to make a selection of several movements from one recording session. ${ }^{19}$ The observed $\mathrm{GM}$ s are diagnosed as normal if the movements are complex, including neck, trunk and limb movements appearing in a variable sequence as fluent, and if their intensity wax and wane. ${ }^{19}$ If the GM s are monotonous, have less complexity and are repetitive in pattern, they are judged as abnormal and as being of 'poor repertoire'. ${ }^{19}$ Other abnormal patterns are the 'cramped-synchronized' type, when the movements are occurring en bloc and generalized muscle contraction and relaxation appear al most simultaneously, or the movements may occur in a jerky and exaggerated manner and in chaotic order. ${ }^{19}$ If GM s are very short then they are difficult for judgment defined as 'hypokinesia'. ${ }^{20}$ Several studies employing this new assessment have been carried out and they showed that the early normal or abnormal findings of the GM quality are highly predictive for later outcome. ${ }^{19}$

A ssessment of GM s is based on the concept of ontogenetic adaptation corresponding to the development of human organism, which is during each developmental stage adapted to the internal and external requirements. ${ }^{19}$ Prechtl stated that spontaneous motility, as the expression of spontaneous neural activity, is the marker of brain's appropriate or disturbed function. ${ }^{13}$ The observation of the unstimulated fetus or infant which is the result of spontaneous behavior without sensory stimulation is the best method to assess its CNS capacity. All endogenously generated movement patterns from an unstimulated CNS could be observed as early as from the 7 to 8 weeks of postmenstrual age, with a reach repertoire of movements developing within the next 2 or 3 weeks, continuing to be present for 5 to 6 months postnatally. ${ }^{8}$

The identification of 'CNS depression' during fetal life is based on precompetences (opening of the eyes, variety of facial expressions), primary reflexes (rhythmical bursts in the sucking pattern) and quality of GM s. ${ }^{19,21}$ The addition of cranial signs (such as insufficienthead growth and overlapping sutures), high arched palate and neurological thumb to neurological fetal signs could be a valuable complement. ${ }^{21} \mathrm{M}$ oreover, the identification of dynamic and static patterns of the symptoms may be as hel pful to date the insult as postnatal. The more stable the signs, the more precise is the timing of the insult. In the presence of neurological signs in fetuses, the next step is to proceed to the clinical synthesis. ${ }^{21}$ In order to do so, all examinees should be followed at least till the age of two years, when their categorization to disabling or nondisabling CP can be possible, based on clinical neurological findings and presence or absence of the ability to walk. ${ }^{21}$

Obstetricians would have a great benefit if it was possible to assess the condition of the fetal nervous system, especially due to the fact that in many cases obstetricians are considered responsible for the brain damage in neonates, regardless of a growing pool of evidence that most of such damages are consequences of prenatal complications. ${ }^{21}$ Even after the fetal brain anatomy can be visualized by ultrasound and the development of the fetal brain is well understood, not much is known about the functional development of the fetal CNS. In other words, the function of fetal CNS is poorly accessible. Observation of fetal behavior provides a direct assessment of brain - the most important human organ. ${ }^{22}$ It is possible to look closely at the functioning of the CNS and the brain. Prenatal motility is considered to reflect the developing nervous system but also involves functional and maturational properties of fetal hemodynamics and the muscular systems. ${ }^{22}$ Prechtl et al have explored spontaneous motility during human development. ${ }^{19}$ They introduced the concept of ontogenic adaptation, meaning that during each developmental stage, the functional organization has to take into account internal and external requirements. ${ }^{19}$ A ny fetal brain damage will interfere with endogenous motor activity. Therefore, spontaneous movements, as an expression of neural activity, could be used as a marker for fetal brain status. ${ }^{19}$ Consequently, the observation of the unstimulated fetus or infant should contribute significantly to the assessment of CNS function. Even after delivery, behavioral patterns frequently provide the most useful indicators of brain function in spite of having extending access to the neurological, physiological and pharmacological measures. ${ }^{22} \mathrm{~T}$ his remarkable continuity of endogenously generated activity from prenatal to postnatal life may allow identification of fetuses and infants with emerging neurological impairment.

During 40 weeks of gestation, the repertoire of fetal activities constantly expands, correlating precisely with structural development of the CNS. ${ }^{23} \mathrm{M}$ ajor developmental events, such as the establishment of neural connections in the different regions of the brain, are accompanied by the occurrence of new patterns of fetal activity or with the transformation of the existing patterns. ${ }^{23}$ The organization of behavioral states during the final weeks of pregnancy shows that the connection between cerebral cortex and periphery is established, and that the cerebral cortex takes control over fetal activity. This also indicates the ability of the fetus to perceive and process external signals. Furthermore, the latest results indicate that even higher brain functions, such as learning, develop in utero during the last weeks of gestation. ${ }^{23}$

The major problem with the study of fetal behavior is that it is very time-consuming and not enough functional for routine clinical practice. In order to overcome the issue of subjectivity fetal assessment should be recorded. Only if normal behavior is fairly understood, it is possible to identify and to recognize abnormal behavior before birth. ${ }^{6,24}$

First report on fetal behavior obviously suggested that these studies should be standardized as much as possible. A n objective analysis with strict application techniques and the use of valid reference ranges appropriate for the gestational age are essential. ${ }^{11}$ Without such standardization, comparisons with preceding or subsequent fetal measurements and comparable assessments cannot be made. In order to achieve this goal, the 
first study describing the 4D sonographic techniques used for obtaining longitudinal standard parameters of fetal neurological development in all trimesters of a normal pregnancy has been published. ${ }^{25} \mathrm{M}$ easurement of seven parameters in the $1 \mathrm{st}$ trimester and 11 parameters in the 2nd and the 3rd trimesters correlated with gestational age. Those parameters have been followed longitudinally through all trimesters and showed increasing frequency of fetal movements during the first trimester. A tendency toward decreased frequency of facial expressions and movement patterns with increasing gestational age from the 2 nd to the 3 rd trimesters has been confirmed. ${ }^{25}$

Despite the longstanding conclusion that it is possible to make valid conclusion about brain function from observed movement patterns and no generalized antenatal behavior, the antenatal screening of fetal behavior has not been devel oped to identify fetuses with probable CNS defects. Recent study from M orokuma tried to produce screening test that would be less time-consuming and probably cost-effective as compared with their previous study. ${ }^{26}$ They developed a brief ultrasound examination to distinguish fetuses with compromised CNS function from the general population and compared it with their study. ${ }^{26}$

Improvements in technology and procedures enabling direct access to the fetus in utero, are generating the impetus for prenatal developmental research to move beyond the simple documentation that behavior abnormalities during pregnancy can produce apparent effects in their offspring after birth. R ather, we emphasize an increasing need to focus on how prenatal events affect the fetus, its behavior and its relationship with environmental conditions in utero. Investigation of behavioral potentials of the fetus will endorse understanding of the mechanisms of normal and abnormal development leading to the predictable behavioral outcomes after birth. In other words, behavioral study of the fetus will be necessary to understand the origins of motor and sensory capabilities of infants and the mechanisms of altered devel opmental outcomes. ${ }^{27}$

A wareness of changing risks and impending of significant neurodevelopmental problem is an essential principle of perinatal medicine. Unfortunately, adverse neurological outcomes can develop after failure to timely recognize CNS developing risk and timely referral of individuals to the appropriately staffed facility. The timing of referral can be critical, but there are obvious difficulties which may result in late referral or even in denial of referral.

\section{Fetal Origin of Neonatal B ehavior}

The existence of motoric competence of the preterm and even term new born infants raises an important issue of the origins of behavior in prenatal life. The continuity of the maturation process from the fetus to the neonate all ows speculating on the predictive value of optimal and nonoptimal neurological functions as observed in the fetuses and their morphological consequences. Neonatologists know that early prediction concerning outcome is reliable only at the two ends of the spectrum, e.g. optimal and very abnormal situations. However, in intermediate situations the quality of observations achieved by 3D/4D US already allows to demonstrate the prenatal onset of brain damage, based on morphol ogic and functional signs. ${ }^{26,27}$ Their identification during the second half of pregnancy may serve as a retrospective marker of a prenatal insult.

In order to investigate the continuity of behavior, fetal movements were recorded by 4D US and after birth neonatal spontaneous motor activity was recorded by video camera. ${ }^{28}$ There were no movements observed in fetuses which were not present in neonates, while Moro reflex was present only postnatally. Fetal motor activities seem to indicate typical features as regards regulatory behaviors in the first month, and they are the starting point for individual differences in reactivity and regulation in infancy. ${ }^{29}$ Fetal condition reflects the stage of growth of the CNS and is a stable individual characteristic indicating the postnatal state organization. ${ }^{30,31}$ For example, the character of $\mathrm{GM}$ s remains essentially unchanged from their emergence until the second month after birth at term. During the first postnatal weeks, i.e. after suspension of intrauterine movement restriction, the slow speed and small amplitude of general movements are still observed, suggesting a carry-over effect from the prenatal to the postnatal period. Fetal movement patterns might reflect the emotional state of the fetus. ${ }^{30}$

V ery important investigation of definitely abnormal GM in the general population showed positive predictive value for neurodevel opmental impairment of $18 / 100$ and for CP 12/100, while negative predictive value was $100 \%$. $^{32}$ The authors concluded that the good predictive value of $\mathrm{GM}$ assessment in high-risk populations cannot be generalized to the general population. ${ }^{32}$

\section{Cerebral Palsy and Perinatal Period}

The traditional concept that brain damage is caused during birth or early neonatal period has been challenged with antenatal inflammatory and unclassifiable factors, which recently are considered as the most important etiologic factors. ${ }^{33-36} \mathrm{CP}$ is an 'umbrella' term for disorders of development, movement and posture, resulting in limitations of activity due to nonprogressive impairment of developing brain. ${ }^{33-35}$ The motor disorders in CP are often accompanied by disturbances of sensation, cognition, communication, perception and/or behavior, and/or by a seizure disorder. 'A ttributed to' is purposely vague because understanding of developmental neurobiology is evolving rapidly. 'Disturbances' is used as a comprehensive term referring to 'events or processes that in some way interrupt damage or otherwise influence the expected pattern of brain maturation' . 37,38 Those events or processes are many, with consequences varying from very conspicuous to very subtle. The worldwide preval ence is estimated to be from 1.5 to 3.0 per 1000 live birth in developed countries, which did not significantly change for many decades, respectively. ${ }^{39,40}$ Improvement of obstetrical and neonatal care did not result in 
decreasing prevalence rate of $C P$ in term infants, while in preterm infants below 28 weeks of gestation the prevalence rate significantly decreased. ${ }^{39,41,42}$ The decrease in CP preval ence observed since the 1980 s had ceased. CP is the most common chronic motor disability of childhood. The diagnosis is retrospective and it is rarely made before the age of 6 months when the infant is severely affected. ${ }^{41}$ The specificity of the diagnosis improves as the child ages and the nature of the disability evolves. ${ }^{41} \mathrm{CP}$ does not result from a single event but rather from a sequence of interdependent adverse events. This time frame of evolving adverse events should be taken into account when considering the possibility of $\mathrm{CP}$ diagnosis in infants. ${ }^{41}$ Periventricular white-matter injury is now the most common cause of brain injury in preterm infants and the leading cause of chronic neurological morbidity and $C P .{ }^{41}$ Standardized methods of clinical neurological assessment from the neonatal period onward were developed in order to identify three grades of neurological impairment: severe, moderate and mild. The clinical identification of severely affected patients is less problematic than the identification of moderately and mildly affected infants. In severely affected children, brain damage can be present and then cranial ultrasound, magnetic resonance imaging, magnetic resonance spectroscopy and diffusionweighted imaging of the brain are helpful in very low birth weight, premature and in term infants with encephalopathy. ${ }^{40,41}$ In some affected infants with $\mathrm{CP}$, brain damage could not be diagnosed with available diagnostic imaging methods.

From the pediatric experience, it is well known that one should wait until the age of 6 months postnatally to be able to diagnose a severe $C P, 12$ months for a moderate $C P$ and 24 months for a minor nondisabling CP. This delay for the full clinical expression of functional consequences of a brain damage depends on brain maturation. DiPietro was right in saying that a consensus recognizing the fact that fetal neurobehavior reflects the developing nervous system is emerging. ${ }^{29} \mathrm{~T}$ he purposes of early diagnosis of CP could be important from the point of view of the infant, the mother, the family, and the gynecologist, who is often accused for clinical negligence, as well as pediatrician who is following the infant. Although randomized studies confirming that the early intervention as an effective strategy for treatment of $C P$ is not available, it should be considered as feasible. Because the etiology of CP is mostly shifted toward the prenatal period, attempts were made to diagnose neurological impairment in the prenatal period. ${ }^{25,41}$

One crucial question often posed to neonatologists is to determine the exact timing of brain damage, prenatal or intrapartum, in the context of neonatal encephal opathy. In this perspective, repeated neurological assessments over the first days of life allow identification of two profiles. The first, a dynamic profile, is associated with signs of CNS depression increasing within the first 3 days and then decreasing gradually with obvious improvement in alertness, motor activity and sucking. ${ }^{42}$ This profile is typical of recent insult, most often intrapartum. The second one, a static profile, is disclosed by lack of changes al ong repeated assessments in the first week of life. This latter profile is typical of a prenatal insult that occurred in utero at least several weeks earlier and therefore, already stable at the time of birth. It is estimated that $36 \%$ of cases of $\mathrm{CP}$ is caused by antepartal events, while perinatal/neonatal causes are responsible for another $41 \%$ cases of $C P .{ }^{41}$

\section{Advantages of 4D compared with 2D Ultrasound in the Assessment of Fetal Behavior}

A lthough 2D US enhanced understanding of fetal neurological development, the real improvement in study of fetal behavior has been achieved by introduction of $4 D$ US ${ }^{43}$ 3D US has been available for almost 15 years. During its continuous development, significant improvement has been achieved with different imaging modes like multiplanar imaging, volume rendering, surface rendering, 3D color Doppler and 3D volumetry, cine-loop animation, postprocessing, cutting, tomographic ultrasound imaging, inverse mode, etc. However, the $3 \mathrm{D}$ image freezes the object and therefore does not provide information on movements or any information about the dynamic changes of the object of interest which 4D does. Human eyes are able to differentiate betw een images up to a frequency of about 12 images per second, which means that 4D US machines should have appropriate frame rate with specially designed probes and fast computer rendering device. ${ }^{22,44} \mathrm{At}$ the moment 4D US scanning is not real-time and available machines can reach up to about 20 images per second, depending on volume, size, resolution and the mechanics of the probe. Nevertheless, even at these relatively slow frame rates the ability to study fetal activity is strikingly good. This new diagnostic tool enables the assessment of fetal face and other surface features of the fetus, such as fetal extremities. Study of relatively unexplored area of fetal behavior as a possible measure of neurological maturation is also possible by $4 \mathrm{D} \cup \mathrm{S}^{45}$

In the early 2 nd trimester, 4D US provides simultaneous visualization of all four extremities and enables depiction of isolated arm movements and their direction. ${ }^{44}$ Because of the limitations of 2D US only five types of isolated hand movements can be described. They include: hand to head, hand to trunk, hand to foot, hand to fluid and hand to the uterine wall. If one performs 4D US hand to head movement can be differentiated into seven subgroups: hand to head, hand to mouth, hand near mouth, hand to face, hand near face, hand to eye and hand to ear. ${ }^{44}$

With 4D US, it is now feasible to study a full range of facial expressions, including smiling, crying, scowling, mouthing and eyelid movements. ${ }^{45}$ The observation of facial expression may be of scientific and diagnostic value and such approach opens entirely new field of fetal awareness. Preterm infants may be conscious to a limited degree from about 25 weeks, when the thal amocortical connections are established, while term infant is conscious of her/himself, the mother and her/his body. ${ }^{46} \mathrm{It}$ seems like a new field of 'fetal psychology' has been introduced, thanks to the development of 4D US technology, with the 
speculation that maj or discoveries regarding human neurobehavioral development lay ahead as the field of fetal psychology matures. ${ }^{47}$ The main advantages of new 4D US technique are the ability to study fast fetal movements in the surface-rendered mode. ${ }^{48}$ Its important advantage in comparison with 2D US is the capability to visualize the whole fetus continuously. ${ }^{48}$ Fetal movements such as yawning, swall owing and eyelid movements cannot be displayed simultaneously with 2D US, while with 4D US the simultaneous facial movements can be clearly depicted. 45

Recently, multicentric studies of fetal brain function have been carried out, ${ }^{49,50}$ with the aim to establish the standards of fetal peripheral and body movements, and facial expressions as additional diagnostic criteria for prenatal brain impairment. ${ }^{51}$ We are almost convinced that 4D US will find the place in everyday obstetrical practice, combining patient acceptance and sensitivity of diagnosis. ${ }^{51-54}$

\section{Postnatal Assessment of Neonates after Fetal 4D US Assessment of Neurobehavior}

The A miel-Tison N eurological A ssessment at Term (A TNAT) relies on responses to specific maneuvers and has specific contribution in the exploration of passive and active tone according to neurological maturation. ${ }^{20,55}$ The clinical significance of this type of assessmentwas morefully understood when Sarnat ${ }^{56-58}$ reviewed anatomical and physiological correlates of early neurological devel opment. In fact, it became possible to clinically dissociate the development of upper and lower motor systems: ${ }^{56-58}$

1. The lower system, consisting of the brainstem and cerebellum, matures early (beginning at $24 \mathrm{GW}$ ) in an ascending wave; its essential role is to maintain posture against gravity and flexor tone in the limbs

2. The upper system, consisting of the cerebral hemispheres and basal ganglia, matures later (beginning at $32 \mathrm{GW}$ ) and rapidly for the first 2 years in a descending wave; its essential role is to control the lower system, with relaxation of the limbs and control of the antigravity forces, finally allowing erect posture, walking and fine motor skills.

This distinction became even more relevant for clinicians after pathological and radiological data had shown that brain damage is mainly located in cerebral hemispheres, in the full term infant with hypoxic-ischemic encephalopathy or in the preterm infant with periventricular leukomal acia (PV L ). ${ }^{56-58}$ The ATNAT may be used to determine the neurological status during the first days of life for full-term infants or at $40 \mathrm{GWs}$ of postmenstrual age for premature neonates. In terms of physiological correlates, it is satisfactory to cluster the different items into four subgroups according to their conceptual meaning: A dequate hemispheric growth, absence of CNS depression, integrity of the upper motor control, stability of autonomic nervous system (ANS). ${ }^{21,56,57,58}$ W hen result for each item is normal, it seems reasonable to conclude to CNS optimality. ${ }^{21}$
In addition, the identification of three signs al ready present at birth offers a precious clue to fetal brain damage, when observed in a cluster: $:^{21,56-58}$

1. High-arched palate (due to insufficient molding forces of a hypoactive tongue)

2. N onreducible adduction of the thumb in a clenched fist (due to absence of spontaneous motor activity) and

3. Cranial ridges over each suture or restricted to the squamous suture (due to severe or moderate impairment of hemispheric growth).

Using 3D US, only two of these three signs can be diagnosed in utero. As for now, it remains impossible to visualize the high-arched palate routinely with 3D surface imaging since this technique does not permit simple visual ization of deep structure in the oral cavity. However, detection of the two other signs as a specific expression of brain impairment appears promising.

Development of Prechtl's general movements (GM) for postnatal neurological evaluation encouraged obstetricians to implement this technique for fetal neurological evaluation using 2D ultrasound. ${ }^{12,13,19}$ The fact that the same criteria could be used for the fetus and young infants seemed especial ly attractive. Development of computer and ultrasound technology enabled evaluation of fetal $G M$ in three dimensions and in real time. ${ }^{26,45,50}$ A ssessment of GM includes the consideration of body movements (arms, legs, neck and trunk) spreading in variable sequences with gradual beginning and end. They wax and wane in intensity, force and speed, being fluent and elegant, revealing the complexity and variability of motor activity al ready present even in early stage. GM has to be videotaped and then analyzed based on G estalt perception, which provides an overall impression of GM s with standardized procedures. ${ }^{19,59}$ Subsequently, movement patterns will be described in terms of complexity, variability and fluency. ${ }^{59} \mathrm{GM} \mathrm{s}$ will finally be classified as normal-optimal, normal-suboptimal, mildly abnormal and definitely abnormal. ${ }^{60}$ W hile the application of GM s in postnatal life is standardized and in spite of encouraging results of fetal GMs in the last 25 years, which showed that qualitative assessment of GMs is a good marker of brain dysfunction, they did not shift the diagnosis of neurological impairment to the prenatal period. R ecent studies revealed that longitudinal evaluation of GMs and brain ultrasonography before and after birth in fetuses from pregnancies complicated by hypertensive disorders or preterm labor between 26 and 34 weeks gestational age may provideinsight in the predictive value of fetal GMs for nervous system integrity and neurological outcome. $^{2}$

\section{Kurjak's Antenatal Neurobehavioral Test (KANET)-A New Screening Tool}

A new scoring system for fetal neurobehavior based on prenatal assessment by 3D/4D sonography has been recently introduced. ${ }^{7}$ That scoring system is a combination of some parameters from fetal GM assessment and from postnatal A TNAT signs, which 
can be prenatally easily visualized by $4 \mathrm{D}$ US. ${ }^{21}$ The parameters were chosen based on developmental approach to the neurological assessment and on the theory of central pattern generators of GM emergence. They were the product of multicentric studies conducted during several years which resulted with the most significant parameters for the assessment of fetal neurological devel opment. ${ }^{7,61-65}$ The authors devel oped a three-point scal e for isolated head anteflexion, isolated hand, leg, hand to face and finger movements, while for the assessment of cranial sutures, isolated eye blinking, facial alterations and mouth opening two-point scale were applied. ${ }^{7}$ The distinction between scores 0 and 2 is evident, whereas uncertainty may exist with regards to the assignation of a score of 1 , the latter indicating an abnormal result of moderate degree. The precise description of the moderate abnormal performance is included for each item in the record. ${ }^{7,62}$ Interpretation of total score is given in Table 1.,62

The new scoring test has been produced by assessment of fetuses with optimal neurological findings and severely brain damaged fetuses by comparison of fetal and neonatal findings. ${ }^{7}$ In the group of 100 low-risk pregnancies, the new scoring test has been applied retrospectively. A fter delivery, postnatal neurological assessment (ATNAT) was performed, and all neonates assessed as normal reached a score between 14 and 20 , which was assumed to be a score of optimal neurological development. ${ }^{7,27,61-65}$ Thenew scoring system was then applied in the group of high-risk pregnancies in which, based on postnatal neurological findings, three subgroups of newborns were found: Normal, mildly or moderately abnormal and abnormal. ${ }^{62} \mathrm{~B}$ ased on this, a neurological scoring system has been proposed. All normal fetuses reached a score in the range from 14 to 20 . Fetuses, who were postnatally described as mil dly or moderately abnormal, achieved prenatal score of 5 to 13 , while another ten fetuses postnatally assigned as neurologically abnormal had a prenatal score from 0 to 5 . A mong this group there were fetuses with alobar holoprosencephaly, severe hypertensive hydrocephaly, tanatophoric dysplasia and multiple malformations. ${ }^{62}$

B ased on several years of research the same group of authors has proposed a new test for antenatal application. There is a similarity between neonatal optimality test of A miel-Tison and new scoring system for the assessment of neurological status in fetuses, which is a combination of postnatal ATNAT and GM assessment. ${ }^{7}$ One of the differences was that the analytical criteria of typical passive and active tone in the neonate cannot be elicited in the fetus: Head anteflexion vs retroflexion, ventral vs dorsal incurvations in the axis, both being of the utmost importance postnatal ly to confirm CNS optimality. ${ }^{7,21}$ However, the status of the fetus should be reflected in the typical $\mathrm{GM} \mathrm{s.}{ }^{7}$

$B$ esides in normal fetuses, fetal behavior has been assessed in growth-restricted fetuses before scoring system has been invented, and KANET was studied in low- and high-risk pregnancies. $^{63,64}$
In the prospective study of 50 pregnancies with IUGR fetuses in the 3rd trimester of pregnancy, a tendency of less behavioral activity in IU GR than normal fetuses has been noted. ${ }^{63}$ The results of the study encouraged future investigation of the use of $4 \mathrm{D}$ ultrasound for quantitative and qualitative assessment of fetal behavior as possible indicators of the neurological condition in IU GR fetuses. ${ }^{63}$

In the study of fetal behavior, the scores of KANET were compared in high-risk and in normal pregnancies. ${ }^{62}$ After delivery, the results of KANET from both groups were compared with A TNAT test. Statistically, significant difference between groups for eight out of 10 KANET parameters has been evident. Comparison of KANET and ATNAT showed statistically significant, moderate correlation between the two tests, which means that the neuropediatric exam (ATNAT) confirmed the prenatal findings of $4 D$ US (KANET). The authors concluded that these preliminary results were promising and stated that further studies are needed before the test could be recommended for wider clinical practice. ${ }^{62}$

Their conclusion prompted the publication of the multicentric study with higher number of examinees. ${ }^{64}$ This study included 266 fetuses in four centers with the aim to compare prenatal data after application of KANET test and postnatal data with the application of ATNAT and GMS assessment when appropriate. It was revealed that 234 out of 266 fetuses were neurologically normal, seven abnormal and in 25 borderline results have been obtained. ${ }^{64}$ Out of seven abnormal fetuses A TNA T was borderline in five and abnormal in two, whereas GM assessment was abnormal in five and definitely abnormal in two. Out of 25 KANET borderline fetuses, ATNAT was normal in seven, borderline in 17 and abnormal in one, whereas the GM assessment was as follows: normal-optimal in four, normal-suboptimal in 20, and abnormal in one ${ }^{64}$ In summary, out of 32 borderline and abnormal fetuses ATNAT was normal in seven, borderline in 22 and abnormal in three; GM assessment was normal-optimal in four, normal suboptimal in 20, abnormal in six and definitely abnormal in two.$^{64} \mathrm{On}$ the basis of this preliminary results, it could only be speculated why this happened after delivery. ${ }^{64} \mathrm{It}$ is known that birthing process is affecting neonates neurologically, but it seems like some fetuses got liberated after birth due to numerous intrauterine constrains. ${ }^{64}$

During the period of 1 year, in the prospective longitudinal cohort study, the KANET was applied in 620 singleton pregnancies, between 26 th and 38 th week of gestation. ${ }^{65}$ There were 520 pregnant women in high-risk and 100 pregnant women in low-risk group. Comparison of KA NET scores in low- and high-risk pregnancies was expectedly statistically significant. ${ }^{65}$ The largest incidence of fetuses with abnormal KANET was in the group of fetuses who had siblings with cerebral palsy. The largest incidence of the borderline KANET has been found in the group of fetuses whose mothers had fever during pregnancy. ${ }^{65}$ The following parameters of KA NET test significantly differed between the fetuses from low- and high-risk pregnancies: Overlapping cranial sutures, head circumference, 
isolated eye blinking, facial expressions, mouth movements, isolated hand movements, isolated leg movements, hand to face movement, finger movements and general movements. ${ }^{65}$

\section{Osaka Consensus - The Standardization of KANET and Postnatal Assessment}

The studies of fetal behavior by 4D US have provided us with many useful information concerning the neurological status of the fetus from low- and high-risk pregnancies. ${ }^{52-54,62-65} \mathrm{M}$ any centers have been involved in the investigation which is the reason for the standardization of existing neurological prenatal test and its postnatal evaluation. The consensus statement on the standardization of prenatal and postnatal neurological assessment has been created in 0 saka, J apan at the International
Symposium on Fetal Neurology organized by International A cademy of Perinatal M edicine on October 24, 2010.

The KA NET should be performed in the 3rd trimester from 28 th to 38th week of gestation. The assessment should last from 15 to 20 minutes, and the fetuses should be examined when awake. If the fetus is sleeping, the assessment should be postponed for 30 minutes or for the next day between 14 and 16 hours. In cases of definitely abnormal or borderline score, the test should be repeated every two weeks till delivery. Special attention should be paid to the facial movements and eye blinking, which are prenatally informative and important (the face is the mirror of the brain). The frequency of facial and mouth movements should be 0 to 5 and more than 5 . Overall number of movements should be defined in very active or

Table 1: Proposal for the new KANET assessment tool consisting of eight parameters

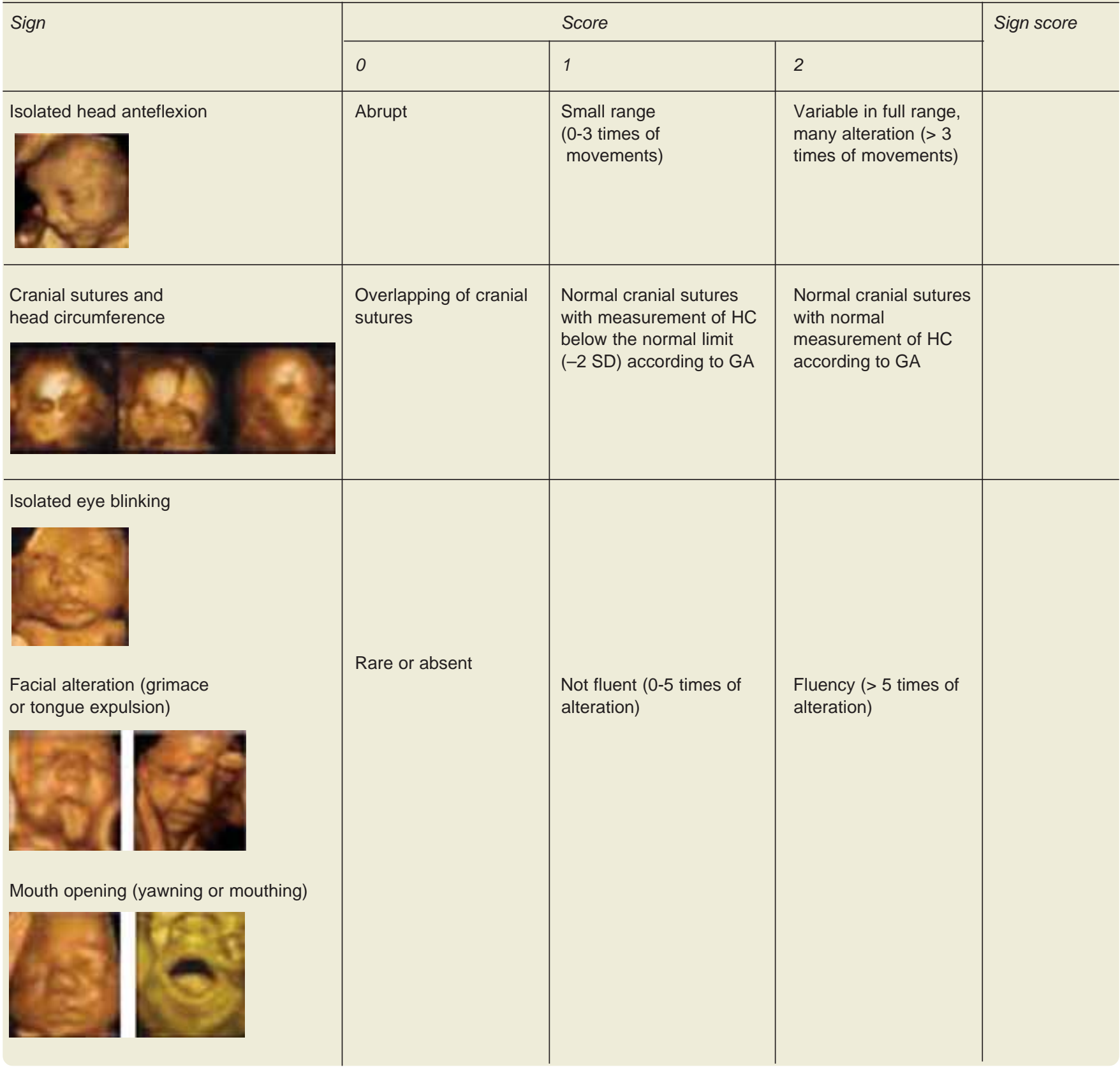


Contd...

\begin{tabular}{|c|c|c|c|c|}
\hline \multirow[t]{2}{*}{ Sign } & \multicolumn{3}{|c|}{ Score } & \multirow[t]{2}{*}{ Sign score } \\
\hline & 0 & 1 & 2 & \\
\hline Isolated hand movement & Cramped & Poor repertoire & Variable and complex & \\
\hline Isolated leg movement & Cramped & Poor repertoire & Variable and complex & \\
\hline Hand to face movements & Abrupt & $\begin{array}{l}\text { Small range (0-5 times of } \\
\text { movement) }\end{array}$ & $\begin{array}{l}\text { Variable in full range, } \\
\text { many alternation ( }>6 \\
\text { times of movements) }\end{array}$ & \\
\hline Fingers movements & $\begin{array}{l}\text { Unilateral or bilateral } \\
\text { clenched fist, } \\
\text { (neurological thumb) }\end{array}$ & $\begin{array}{l}\text { Cramped invariable } \\
\text { finger movements }\end{array}$ & $\begin{array}{l}\text { Smooth and complex, } \\
\text { variable finger } \\
\text { movements }\end{array}$ & \\
\hline Gestalt perception of GMS & Definitely abnormal & Borderline & Normal & \\
\hline & & & Total score & \\
\hline
\end{tabular}

inactive fetuses and compared with normal values from our longitudinal study. ${ }^{50}$

All the investigators should have extensive hands on education for the application of K A NET test in low - and highrisk pregnancies. Interobserver and intraobserver variability should be available.

It is advisable to use 4D US machines with frame rate of at least 24 volumes/seconds.

A ccording to our experience after multicentric studies, the new modified KANET test should be used with eight instead of 10 parameters as follows (see Table 1 ):

- Facial and mouth movements are combined in one category

- Isolated hand movements and hand to face movements are combined in one category.

Table 2: Interpretation of KANET score

\begin{tabular}{|ll|}
\hline \multicolumn{2}{|c|}{ Table 2: Interpretation of KANET score } \\
\hline Total score & Interpretation \\
\hline $0-5$ & Abnormal \\
$6-13$ & Borderline \\
$14-20$ & Normal \\
\hline
\end{tabular}

The score should be the same for abnormal fetuses 0 to 5 , borderline score is from 6 to 13 and normal score is 14 or above (Table 2).

A ntenatal assessment flowchart is presented in the Figure 1.

\section{Postnatal Assessment}

Postnatal assessment of behavior often provides the best indicators of brain function despite having direct access to neurological, physiological and other diagnostic methods. It is even better indicator of brain function in terms of prediction of neurological outcome than neurological assessment al one.

The following groups of newborns should be assessed:

- Gestational age at delivery. Preterm (<37 w eeks of gestation) and term infants (from 37-42 weeks of gestation)

- Eutrophic, hypotrophic and hypertrophic newborns

- Spontaneous vaginal, induced vaginal, vacuum extraction, elective cesarean, emergency cesarean, according to the mode of delivery.

A fter 4D US assessment of behavioral patterns in the fetuses from high-risk pregnancies, it is very important to continue with 


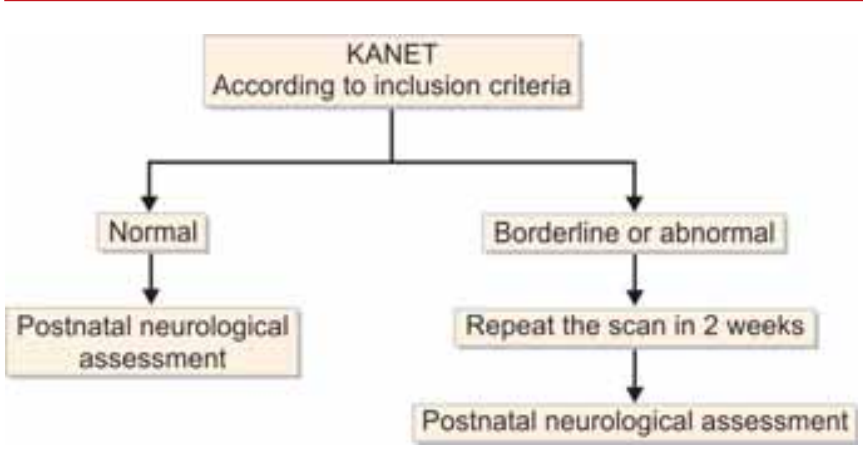

Fig. 1: Antenatal assessment

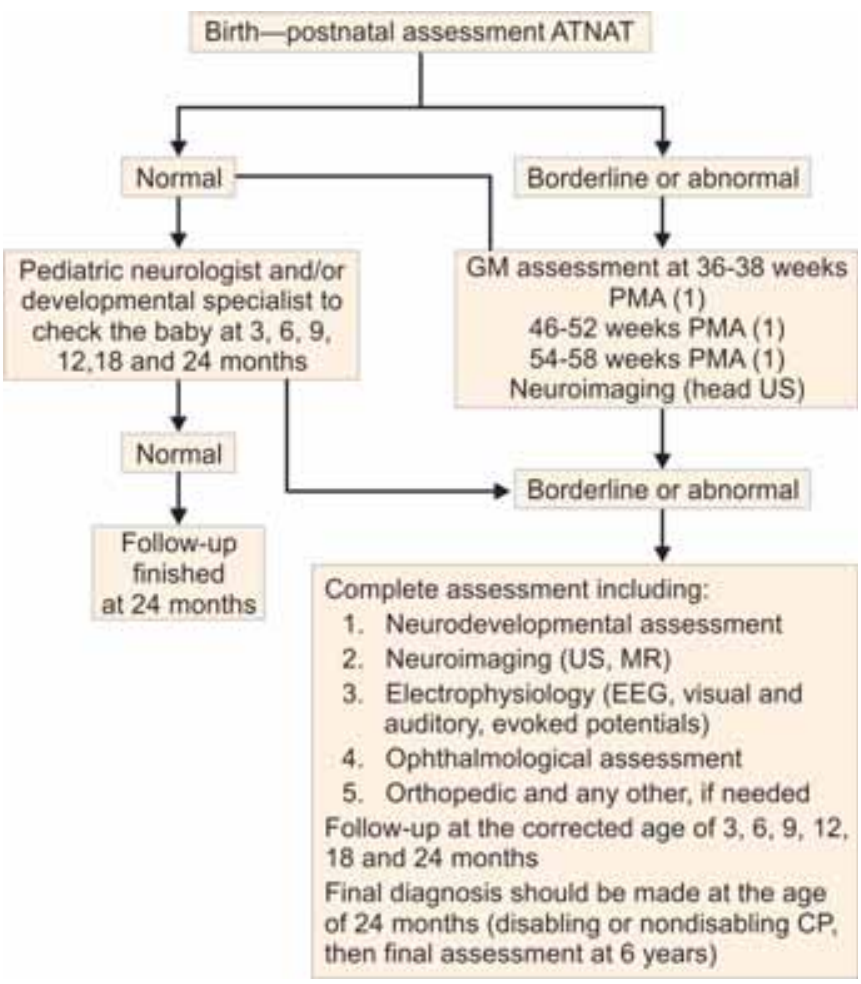

Fig. 2: Postnatal assessment

follow-up after delivery in infants who were borderline or abnormal as fetuses. Postnatal assessment of neonates includes:

1. Initial neurological assessment according to A miel-Tison's methodology (ATNAT) in the early neonatal period and every two w eeks in preterm infants till discharge and at the post-menstrual age (PM A) between 37 and 40 weeks

2. If ATNAT is borderline or abnormal, initial assessment of general movements at the age of 36 to 38 weeks of PM A should be performed than at writhing age (between 46 and 52 weeks), and at the fidgety age after 54 weeks of PM A. If the finding of fidgety movements is mildly abnormal or definitely abnormal, then one more assessment should be done in 2 to 4 weeks till PM A of 58 weeks

3. Brain ultrasonography in the first week of life and every two weeks afterward till discharge. Transcranial Doppler assessment and evoked potentials (visual, auditory, somatosensorial) will be performed, if available. In severely affected infants with grade 3 and above intraventricular
Box 1: Proposal of postnatal magnetic resonance (MR) imaging protocol (Prayer D)

Preferable time: Corrected gestational week $40-44$ or as soon as possible afterward

Field strength 1.5 or $3 \mathrm{~T}$

Sequences:

- T2 turbo spin echo (TSE) (fast spin echo-FSE) with TE 140, 3 section planes, 2-3 $\mathrm{mm}$ slice thickness

- T1 isovoxel $(1 \mathrm{~mm})$ volume sequence

- Axial infrared (IR) or T1

- Axial diffusion tensor-imaging (DTI) or diffusion-weighted imaging (DWI) b-value 700

- Axial susceptibility weighted imaging or T2

Duration approximately 30 minutes

Additional sequences with specific questions:

- In case of suspect tumor or infection: T1 postcontrast

- In case of suspect vascular pathology: Angiography or venous angiography

- In case of suspect ischemic lesions: Perfusion

- In case of suspect metabolic impairment: Single voxel spectroscopy (SVS) (lesion and corresponding region in normal hemisphere) or basal ganglia (in case of screening for lactate) long and short echo time at 1.5T, ultralong and short echotime at 3T

hemorrhage, and those highly suspicious of hypoxic ischemic brain damage, magnetic resonance (M R) should be done, if available. Postnatal magnetic resonance (M R ) protocol proposal by Daniela Prayer is given in the B ox 1 .

4. Infants should be foll owed until the age of at least 24 months when diagnosis of disabling or nondisabling $C P$ can be ultimately made. Infants with CP should be reassessed at the age of 6 years.

The aim of postnatal neurological assessment is early detection of infants at risk for $C P$, undergoing early intervention depending on the type and significance of the disorder. Optimality of CNS development is the major aim of neurological assessment and assessment of $\mathrm{GM}$.

The major aim of the project is to build up a system for analysis of abnormal fetal neurobehavior during pregnancy. Therefore, population examined in this study will be limited to high-risk pregnancy group that will be followed by a prospective study of newborns and infants, compared with the control group. High-risk pregnancies are defined according to Strijbis et al.$^{66}$ $M$ ajor pathologies associated with increased prevalence of $C P$ are as follows: prematurity, antepartum hemorrhage, complications of multiple pregnancies, genetic disorders, intrauerine infections (chorioamnionitis, funisitis, villitis, cytomegal ovirus, toxoplasmosis), intrapartum fever, intrauterine growth restriction, maternal and/or fetal coagulopathy, multiple congenital anomalies, maternal disease (hypothyroidism, diabetes, drug abuse, severe preeclampsia, viral illness), placental pathology (major infarction, thrombotic vasculopathy), tight nuchal cord, childhood causes (a previous child with CP), other causes (fetal hemorrhage, rhesus disease, fetal nonimmunehydrops). 
The basis for this investigation is our own published papers, in which we have demonstrated that by using 4D sonography it is now possible to produce measurable parameters for the assessment of normal neurobehavioral development.

A combined method of standardized neurological assessment proposed by A miel-Tison, neuroimaging intracranial ultrasonography and transcranial color Doppler, cranial M RI, neurophysiology - evoked potentials, polysomnography and general movements assessment at writhing and fidgety age classified according to M ijna Hadders-A Igra et al, ${ }^{8}$ should be used for neurological assessment of newborns and infants.

\section{Postnatal Assessment-Technical Issues}

Pathophysiological considerations of CNS development have been the driving force for successive modifications of the clinical assessment atterm according to A miel-Tison (ATNAT). $M$ ore emphasis has been placed on signs that depend on the integrity of the upper structures (hemispheres), such as axial tone and alertness as well as cranial signs linked to the increase in volume of the cerebral hemispheres. The signs depending on brainstem function (lower structures), such as primitive reflexes and passive tone in limb flexor muscles, have been deemphasized at the neonatal period as they do not provide information about the cerebral hemispheres and basal ganglia. To gain a more precise definition of infant response, a threepoint scale has been devised for each item: 0-normal; 1- moderately abnormal; 2 -abnormal. The scoring system has been standardized for application beginning with the full-term newborn.

In those infants who are scored as moderately abnormal or abnormal after ATNAT, Prechtl's general movements will be assessed. For the postnatal examination, video camera is installed high above the infant who is lying supine in the incubator or bed. Recordings are made while the infant is in state of al ertness 3 and last betw een 30 and 60 minutes in order to collect sufficient number of GM s. L ater on, recordings are viewed at high speed and GM s are then appraised on a visual 'Gestalt perception' of the spatial and temporal variation of motor patterns, with the assessment of their complexity, variability and fluency. The GM s are scored as normal-optimal, normal-suboptimal, mildly abnormal or abnormal. Those who will be scored as mildly abnormal or abnormal the follow-up will be continuously performed at writhing and fidgety age. Multidisciplinary follow-up of infants will be organized according to local programs and possibilities for the follow-up of infants at high neurological risk. Postnatal assessment flowchart is presented in the Figure 2.

\section{REFERENCES}

1. de V ries JI, Fong BF. Normal fetal motility: An overview. Ultrasound Obstet Gynecol 2006;27:701-11.

2. Rosier-van Dunné FM, van Wezel-M eijler G, Bakker MP, Odendaal $\mathrm{HJ}$, de V ries JI. Fetal general movements and brain sonography in a population at risk for preterm birth. E arly Hum Dev 2010;86:107-11.
3. de V ries JI, Fong BF. Changes in fetal motility as a result of congenital disorders: A n overview. U Itrasound Obstet Gynecol 2007;29:590-99.

4. Prechtl HFR. Qualitative changes of spontaneous movements in fetus and preterm infant are a marker of neurological dysfunction. Early Hum Dev 1990;23:151-58.

5. Kurjak A, V ecek N, Hafner T, B ozek T, Funduk-Kurjak B, Ujevic B. Prenatal diagnosis: What does four-dimensional ultrasound add? J Perinat M ed 2002;30:57-62.

6. Kurjak A, Carrera J, M edic M, A zumendi G, A ndonotopo W, Stanojevic $M$. The antenatal development of fetal behavioral patterns assessed by four-dimensional sonography. J M atern Fetal Neonatal M ed 2005;17:401-16.

7. Kurjak A, M iskovic B, Stanojevic M, A miel-Tison C, A hmed B, A zumendi G, V asilj O, et al. N ew scoring system for fetal neurobehavior assessed by three- and four-dimensional sonography. J Perinat Med 2008;36:73-81.

8. Lüchinger $A B, H$ adders-Algra $M$, van $K$ an $C M$, de V ries JI. Fetal onset of general movements. Pediatr Res 2008;63: 191-95.

9. M aeda K. Quantitative studies on fetal actocardiogram. Croat Med J 2005;46:792-96.

10. Prechtl HFR, Einspieler C. Is neurological assessment of the fetus possible? Eur J Obstet Gynecol 1997;75:81-84.

11. Nijhuis JG (Ed). Fetal behaviour: Developmental and perinatal aspects. Oxford: Oxford U niversity Press 1992.

12. de V ries JIP, V isser GH, Prechtl HF. The emergence of fetal behavior (I). Qualitative aspect. Early Hum Dev 1982;7: 301-22.

13. de V ries JIP, V isser GHA, Prechtl HFR. The emergence of fetal behaviour (II). Quantitative aspects. Early Hum Dev 1985;12: 99-120.

14. Rennie JM, Hagmann CF, Robertson NJ. Outcome after intrapartum hypoxic ischaemia at term. Semin Fetal N eonatal M ed 2007;12:398-407.

15. O'Shea TM . Diagnosis, treatment, and prevention of cerebral palsy. Clin Obstet Gynecol 2008;51:816-28.

16. L ongo $M$, Hankins GD. D efining cerebral palsy: Pathogenesis, pathophysiology and new intervention. $M$ inerva Ginecol 2009;61:421-29.

17. Einspieler C, Prechtl HFR, Ferrari F, Cioni G. Prechtl's method on the qualitative assessment of general movements in preterm, term, and young infants. London: M ac K eith Press 2004.

18. Visser GH, Mulder EJ, Tessa Ververs FF. Fetal behavioral teratology. J Matern Fetal Neonatal M ed 2010;23(Suppl 3): 14-16.

19. A raki M, Nishitani S, U shimaru K, M asuzaki H, O ishi K, Shinohara K. Fetal response to induced maternal emotions. J Physiol Sci 2010;60:213-20.

20. Bayat A, Petersen A, Møller M, A ndersen G, Ebbesen F. Incidence of fetal akinesia-hypokinesia deformation sequence: A population-based study. A cta Paediatr 2009;98:3-4.

21. A miel-Tison C, Gosselin J, K urjak A. N eurosonography in the second half of fetal life: A neonatologist's point of view. J Perinat Med 2006;34:437-46.

22. Andonotopo W, Stanojevic M, K urjak A, A zumendi G, Carrera $J M$. A ssessment of fetal behavior and general movements by four-dimensional sonography. Ultrasound Rev Obstet Gynecol 2004;4:103-14.

23. Salihagic-K adic A, M edic M, Kurjak A, Andonotopo W, Azumendi G, Hafner T, Milenkovic D. Four-dimensional sonography in the assessment of fetal functional neurodevelopment and behavioral patterns. U Itrasound Rev Obstet Gynecol 2005;5:154-68. 
24. Azumendi G, Kurjak A. Three-dimensional and fourdimensional sonography in the study of the fetal face. Ultrasound Rev Obstet Gynecol 2003;3:1-10.

25. Kurjak A, Stanojevic M, A ndonotopo W, Scazzocchio-Duenas E, A zumendi G, Carrera J M . Fetal behavior assessed in all three trimesters of normal pregnancy by four-dimensional ultrasonography. Croat M ed J 2005;46:772-80.

26. M orokuma S, Fukushima $K, Y$ umoto $Y, U$ chimura $M, F$ ujiwara $A, M$ atsumoto $M$, et al. Simplified ultrasound screening for fetal brain function based on behavioral pattern. Early Hum Dev 2007;83:177-81.

27. A hmed B, K urjak A, A ndonotopo W, K henyab N, Saleh N, A I$M$ ansoori Z. Fetal behavioral and structural abnormalities in high-risk fetuses assessed by 4D sonography. The Ultrasound Review of Obstetrics and Gynecology 2005;5:1-13.

28. Kurjak A, Stanojevic M, A ndonotopo W, Salihagic-Kadic A, Carrera J M , A zumendi G. B ehavioral pattern continuity from prenatal to postnatal life- a study by four-dimensional (4D) ultrasonography. J Perinat M ed 2004;32:346-53.

29. DiPietro JA, B ronstein $M$ H, Costigan K A, et al. W hat does fetal movement predict about behavior during the first two years of life? Dev Psychobiol 2002;40:358-71.

30. A rabin $B, B$ os $R$, Rijlardsdam $R$, et al. The onset of interhuman contacts. Longitudinal ultrasound observations in twin pregnancies. Ultrasound Obstet Gynecol 1996;8:166-73.

31. DiPietro JA, Costigan KA, Pressman EK. Fetal state concordance predicts infant state regulation. Early Hum Dev 2002;68:1-13.

32. B ouwstra $H$, Dijk-Stigter $G R, G$ rooten $H M$, J anssen-Plas FE, Koopmans AJ, M ulder CD, et al. Predictive value of definitely abnormal general movements in the general population. Dev Med Child Neurol 2010;52:456-61.

33. Shatrov JG, Birch SC, Lam LT, Quinlivan JA, M clntyre $S$, $M$ endz GL. Chorioamnionitis and cerebral palsy: A metaanalysis. Obstet Gynecol 2010;116(2 Pt 1):387-92.

34. Bax M, Goldstein M, Rosenbaum P, Leviton A, Paneth N, $D$ an $B$ et al. Proposed definition and classification of cerebral palsy, A pril 2005. Dev M ed Child N eurol 2005;47:571-76.

35. Rosenbaum $P$, Paneth $N$, L eviton A, Goldstein $M, B$ ax $M$, Damiano $D$, et al. A report: The definition and classification of cerebral palsy. Dev M ed Child Neurol 2007;109:8-14.

36. Palmer FB. Strategies for the early diagnosis of cerebral palsy. J Pediatr 2004;145:S8-11.

37. Morns C. Definition and classification of cerebral palsy: A historical perspective. Dev M ed C hild N eurol 2007;49:3-7. DOI: 10.1111/j.1469-8749.2007.tb12609.x (accessed Dec 12, 2010).

38. Cans C, Dolk H, Platt M, Colver A, Prasauskiene A, Rägeloh$M$ ann IK. Recommendations from the SCPE collaborative group for defining and classifying cerebral palsy. Dev M ed Child Neurol 2007;49:35-38. DOI: 10.1111/j.1469-8749.2007. tb12626.x (accessed Dec 12, 2010).

39. Blair E. E pidemiology of the cerebral palsies. Orthop C lin N orth A m 2010;41:441-55.

40. M cM anus V, Guillem P, Surman G, Cans C. SCPE work, standardization and definition: A $n$ overview of the activities of SCPE: A collaboration of European CP registers. Zhongguo Dang Dai Er Ke Za Zhi 2006;8:261-65.

41. Himmelmann $K, H$ agberg $G$, U vebrant $P$. The changing panorama of cerebral palsy in Sweden (X). Prevalence and origin in the birth-year period 1999-2002. A cta Paediatr 2010;99: 1337-43.

42. Surman G, Hemming K, Platt M J, Parkes J , G reen A, H utton J, et al. Children with cerebral palsy: Severity and trends over time. Paediatr Perinat E pidemiol 2009;23:513-21.

43. Campbell S. 4D or not 4D: That is the question. Ultrasound Obstet Gynecol 2002;19:1-4.

44. Kurjak A, A zumendi G, V ecek N, K upesic S, Solak M, V arga $D$, et al. Fetal hand movements and facial expression in normal pregnancy studied by four-dimensional sonography. J Perinat M ed 2003;31:496-508.

45. Kurjak A, Stanojevic M, A zumendi G, C arrera J M. The potential of four-dimensional (4D) ultrasonography in the assessment of fetal awareness. J Perinat M ed 2005;33:46-53.

46. Lagercrantz $H, C$ hangeux JP. Basic consciousness of the newborn. Semin Perinatol 2010;34:201-06.

47. Eugene KE. A womb with a view: U Itrasound for evaluation of fetal neurobehavioral development. Infant and Child Development 2010;19:119-24.

48. Kurjak A, M iskovic B, A ndonotopo W, Stanojevic M , A zumendi $G, V$ rcic $H$. How useful is $3 D$ and $4 D$ ultrasound in perinatal medicine? J Perinat M ed 2007;3:10-27.

49. Y igiter AB, Kavak ZN. Normal standards of fetal behavior assessed by four-dimensional sonography. J M atern Fetal Neonatal M ed 2006;19:707-21.

50. Kurjak A, A ndonotopo W, Hafner $T$, Salihagic Kadic A, Stanojevic M, A zumendi $G$, et al. Normal standards for fetal neurobehavioral developments- longitudinal quantification by four-dimensional sonography. J Perinat M ed 2006;34:56-65.

51. Hata $T, K$ anenishi $K$, A kiyama $M$, Tanaka H, Kimura K. Realtime 3D sonographic observation of fetal facial expression. J Obstet Gynaecol Res 2005;3:337-40.

52. Hata T, Dai SY, M arumo G. Ultrasound for evaluation of fetal neurobehavioural development: From 2D to 4D ultrasound. Inf Child Dev 2010;19: 99-118.

53. Hata T, Kanenishi K, Sasaki M . Four-dimensional sonographic assessment of fetal movement in the late first trimester. Int J Gynaecol Obstet 2010;109:190-93.

54. Sasaki M, Y anagihara $T, N$ aitoh $N$, H ata T. Four-dimensional sonographic assessment of inter-twin contact late in the first trimester. Int J Gynaecol O bstet 2010;108:104-07.

55. A miel-Tison $C$. Neurological assessment of the neonate revisited: A personal view. Dev Med Child Neurol 1990;32: 1109-13.

56. A miel-Tison C, Gosselin J, G ahagan S. Why is the neurological examination so badly neglected in early childhood? Pediatrics 2005;116:1047.

57. Gosselin J, Gahagan S, A miel-Tison C. The A miel-Tison neurological assessment at term: Conceptual and methodological continuity in the course of follow-up. M ent R etard Dev Disabil Res Rev 2005;11:34-51.

58. Simard M N, Lambert J, Lachance C, A udibert F, Gosselin J. Interexaminer reliability of Amiel-Tison neurological assessments. Pediatr N eurol 2009;41:347-52.

59. Hadders-A Igra M . V ariation and variability: K eyw ords in human motor development. Phys Ther 2010;90:1823-37.

60. Hadders-A Igra M. Putative neural substrate of normal and abnormal general movements. Neurosci Biobehav Rev 2007;31:1181-90.

61. Kurjak A, Tikvica A, Stanojevic M, M iskovic B, A hmed B, A zumendi $G$, et al. The assessment of fetal neurobehavior by three-dimensional and four-dimensional ultrasound. J Matern Fetal Neonatal M ed 2008;21:675-84. 
62. M iskovic B, V asilj O, Stanojevic M, Ivanković D, K erner M, Tikvica A. The comparison of fetal behavior in high risk and normal pregnancies assessed by four-dimensional ultrasound. J M atern Fetal N eonatal M ed 2010;23:1461-67.

63. Andonotopo $\mathrm{W}, \mathrm{K}$ urjak $\mathrm{A}$. The assessment of fetal behavior of growth restricted fetuses by $4 \mathrm{D}$ sonography. J Perinat $\mathrm{Med}$ 2006;34:471-78.

64. Kurjak $A, A$ bo-Y aqoub S, Stanojevic $M, Y$ igiter $A B, V$ asilj $O$, Lebit $D$, et al. The potential of $4 D$ sonography in the assessment of fetal neurobehavior-multicentric study in high-risk pregnancies. J Perinat M ed 2010;38:77-82.

65. Talic A, K urjak A, A hmed B, Stanojevic $M$, Predojevic $M$, Salihagic Kadic $A$, et al. The potential of 4D sonography in the assessment of fetal behavior in high-risk pregnancies. J M atern Fetal N eonatal M ed 2010 (in press).

66. Strijbis EMM , Oudman I, van Essen $P, M$ acL ennan $A H$. Cerebral palsy and the application of the international criteria for acute intrapartum hypoxia. Obstet Gynecol 2006;107: 1357-65. 